\title{
Die Minsker Gruppe der OSZE: Die Lehren der Vergangenheit und zukünftige Aufgaben
}

\author{
Philip Remler, Richard Giragosian, Marina Lorenzini, Sergei Rastoltsev"
}

\section{Zusammenfassung}

Der internationalen Gemeinschaft, vertreten durch die Minsker Gruppe der OSZE, ist es nicht gelungen, die führenden Kräfte in Armenien und Aserbaidschan dazu zu bewegen, den Konflikt um Karabach zu lösen, der 1988 begann und im September 2020 einen Ausbruch neuer Kampfhandlungen erlebte. Auf beiden Seiten waren Anführer und Bevölkerung zunehmend unnachgiebiger geworden; jeder Anführer, der sich kompromissbereit zeigte, lief Gefahr, als Verräter gebrandmarkt zu werden. Die Kämpfe im Jahr 2020 veränderten die Lage vor Ort drastisch. Mit Unterstützung der Türkei gewann Aserbaidschan einen großen Teil des Territoriums zurück, das es eine Generation zuvor verloren hatte. Allerdings musste Aserbaidschan Russland die Entsendung einer großen Friedenstruppe gestatten, die das Land 25 Jahre lang verweigert hatte. Trotz ihrer geschwundenen Autorität kann die Minsker Gruppe zukünftig dazu beitragen, das Vertrauen und die Kommunikation zwischen den Parteien wiederherzustellen und Grenzen zu öffnen, um schließlich Verhandlungen über den künftigen Status der Region zu leiten.

\section{Schlagworte}

Karabach, Aserbaidschan, Armenien, Russland, Türkei, OSZE

Bitte zitieren als: Philip Remler, Richard Giragosian, Marina Lorenzini, Sergei Rastoltsev, Die Minsker Gruppe der OSZE: Die Lehren der Vergangenheit und zukünftige Aufgaben, OSCE Insights 6 (Baden-Baden: Nomos, 2020), https://doi.org/10.5771/9783748911630-06

\section{Einleitung 1}

Die Kampfhandlungen, die am 27. September 2020 zwischen Aserbaidschan und Armenien ausbrachen, erinnerten

* Philip Remler (Hauptverfasser)

Carnegie Endowment for International Peace

Dr. Richard Giragosian

Regional Studies Center, Yerevan

director@regional-studies.org die Welt einmal mehr daran, dass der Konflikt um Karabach sich seit nunmehr 30 Jahren einer Lösung entzieht. ${ }^{2}$ Die Minsker Gruppe der OSZE ist seit 1992 die mit dem offiziellen Verhandlungs-

Marina Dolcetta Lorenzini, M.A.

The Fletcher School, Tufts University

marina.lorenzini@tufts.edu

Sergei Rastoltsev

IMEMO, Moscow

sergej-ras@yandex.ru 
mandat betraute internationale Gruppe. Während dieser Zeit leitete sie wichtige Verhandlungen, konnte allerdings die Anführer von Aserbaidschan und Armenien nicht dazu bringen, die für einen Frieden notwendigen gegenseitigen $\mathrm{Zu}-$ geständnisse einzugehen. Die intensiven Kampfhandlungen der letzten Monate haben die Situation vor Ort verschärft, den Handlungsspielraum der Minsker Gruppe verkleinert und ihre künftige Arbeit infrage gestellt. Dieser Bericht sucht nach Gründen für Fehler in der Vergangenheit und bewertet die Aussichten für eine künftige Rolle.

Der erste der folgenden vier Abschnitte untersucht die Strategien der Krieg führenden Parteien während der Verhandlungen innerhalb der Minsker Gruppe zwischen dem Waffenstillstand von 1994 und dem erneuten Ausbruch der Kriegshandlungen am 27. September 2020. Der zweite Abschnitt analysiert die Bemühungen der Minsker Gruppe während dieser Zeit. Der dritte untersucht die Ziele und Reaktionen der Hauptakteure seit dem 27. September. Der vierte Abschnitt nennt einige Einflussgrößen, welche die weiteren Entwicklungen bestimmen werden.

\section{Verhandlungsstrategien nach dem Waffenstillstand von 1994}

Um zu verstehen, warum Aserbaidschan am 27. September 2020 eine Offensive lancierte, müssen wir die jeweiligen Ziele der Kriegsparteien in den Kampfhandlungen der frühen 1990er-Jahre sowie ihre Ziele seit dem Waffenstillstand verstehen.

\section{Armenien}

Ursprünglich war das Ziel der KarabachBewegung „miatsum“ - die Vereinigung von Nagornyy Karabach mit der Sowjetrepublik Armenien auf dem Wege eines offiziellen Transfers von der Sowjetrepublik Aserbaidschan. Nach dem Zusammenbruch der Sowjetunion wurde formal das neue Ziel der Unabhängigkeit von Aserbaidschan ausgegeben, obwohl der Wunsch nach einer Vereinigung mit Armenien bestehen blieb. In den Kämpfen, die 1988 begannen und sich bis 1992 zu militärischen Operationen in großem Maßstab ausgewachsen hatten, waren die armenischen Streitkräfte siegreich. Armenien vertrieb die aserbaidschanischen Streitkräfte aus Nagornyy Karabach, nahm Şuşa ein (über Jahrhunderte das Bollwerk der aserbaidschanischen Macht in Karabach), besetzte eine Pufferzone rund um das Gebiet, vertrieb die Bewohner*innen und wehrte aserbaidschanische Gegenangriffe $a b$. So erreichte Nagornyy Karabach eine De-facto-Abspaltung, obwohl der Waffenstillstand von 1994 (die Bischkek-Protokolle) keine politische Einigung brachte. Die Resolutionen des Sicherheitsrats der Vereinten Nationen (UN) betrachten Karabach nach wie vor als Teil von Aserbaidschan. ${ }^{3}$

Die Gebiete, die rund um Karabach von armenischen Streitkräften eingenommen wurden, lassen sich in drei Kategorien unterteilen: 
Provinzen zwischen Nagornyy Karabach und Armenien: Lachin und Kelbajar bildeten in den 1920er-Jahren die sowjetische Provinz Rotes Kurdistan. Lachin wurde im Mai 1992 eingenommen, nur Tage nach der Besetzung von Şuşa. Kelbajar wurde während einer Operation im März und April 1993 eingenommen. Aufgrund der Kämpfe waren die kurdische und die aserbaidschanische Bevölkerung gezwungen zu fliehen. Armenien und Nagornyy Karabach betrachteten diese Provinzen als existenziell wichtig, da sie die Landverbindung zwischen ihnen bildeten.

Provinzen zwischen Nagornyy Karabach und Iran: Der Fall von Kelbajar führte zur Revolution in Aserbaidschan. Armenische Streitkräfte lancierten im Sommer 1993 eine Offensive, in deren Verlauf die Provinzen Qubadli, Zangilan und Jabryil eingenommen und die jeweiligen Bewohner*innen vertrieben wurden. Dieses Gebiet, südlich von Karabach bis zum Fluß Aras, grenzt an den Iran; es wurde als strategisch wichtig eingestuft.

Provinzen östlich von Nagornyy Karabach: Ebenfalls im Sommer 1993 nahmen armenische Streitkräfte die strategisch wichtige Stadt Agdam sowie Teile der Provinz Füzuli ein. Die Städte wurden auf der Suche nach Baumaterial geplündert; es verblieb nur wenig Infrastruktur, außerdem wurden die Frontlinien vermint, um einen aserbaidschanischen Gegenangriff zu verhindern. Diese Provinzen galten als die am leichtesten erworbenen und günstigsten Faustpfänder der armenischen Seite.

Das Ziel der armenischen Seite bestand darin, so viele dieser eroberten Gebiete wie möglich zu behalten. Es entstand eine Kluft zwischen der Strategie des damaligen Präsidenten Levon TerPetrosyan und derjenigen der HardlinerGruppe in Nagornyy Karabach und Armenien. Ter-Petrosyan und seine Anhänger*innen glaubten, ein Erfolg könne nur durch den Tausch einiger von Armenien besetzter Provinzen gegen ein Friedensabkommen gesichert werden, welches die Sicherheit und den Status von $\mathrm{Na}-$ gornyy Karabach auch gegenüber einem zukünftig potenziell reicheren und militärisch überlegenen Aserbaidschan gewährleisten würde. ${ }^{4}$ Die Hardliner-Gruppe glaubte, Armenien brauche keine $\mathrm{Zu}$ geständnisse zu machen. Seine Aufgabe sei es vielmehr, den Status quo zu erhalten und auf eine Hinhaltetaktik zu setzen, bis die internationale Gemeinschaft und Aserbaidschan die Unabhängigkeit Nagornyy Karabachs anerkannt hätten.

Dieses interne Zerwürfnis erschwerte für die armenischen Anführer die Verhandlungen mit Aserbaidschan. Große Teile der Bevölkerung sympathisierten mit der Hardliner-Gruppe und waren zunehmend gegen Kompromisse. Anschläge und Androhungen von Gewalt behinderten Kompromissbemühungen und sabotierten Abmachungen, auf die sich die Anführer geeinigt hatten. ${ }^{5}$ Armeniens Premierminister Nikol Pashinyan gestand nach der Unterzeichnung des Waffenstillstands am 9. November 2020: ,[...] als ich dieses Dokument unterzeichnete, war mir klar, dass mir der Tod drohte, nicht nur in politischer, sondern auch in physischer Hinsicht."6 Im Jahr 1999 stürmten Bewaffnete das Parlament, ermordeten höhere Regierungsvertreter*innen und wichtige Abgeordnete"innen 
und machten dadurch das Scheitern jeglichen Friedensplans unvermeidlich. In der Folge spielten die armenischen Verhandlungsführer auf Zeit, in der Hoffnung, die internationale Gemeinschaft würde früher oder später die faktische Situation auch rechtlich anerkennen.

\section{Aserbaidschan}

Die aserbaidschanische Führung war mit dem umgekehrten Dilemma konfrontiert. Während Armenien danach trachtete, die Verhandlungen in die Länge $\mathrm{zu}$ ziehen, bis die Tatsachen vor Ort rechtlich anerkannt wären, wollte Aserbaidschan diese Tatsachen ändern, um sicherzustellen, dass die bestehende Lage niemals international anerkannt werde. Genau wie seine armenischen Gegenspieler fürchtete der derzeitige Präsident Ilham Alijev um die innenpolitische Stabilität, falls er bei den Gebietsansprüchen von Maximalpositionen abrücken sollte.

Der direkteste Weg, die Tatsachen vor Ort zu verändern, war mit Waffengewalt. Mit diesem Ziel stellte Aserbaidschan konstant erhebliche Geldmittel für das Militär zur Verfügung. Eine andere Strategie der Veränderung war die Mobilisierung der internationalen Gemeinschaft, um bei Verhandlungen Druck auf Armenien auszuüben und Zugeständnisse zu erzwingen. In den 1990er-Jahren hofften die Aserbaidschaner, ihre Öl- und Gasvorkommen würden den Westen dazu veranlassen, Armenien unter Druck zu setzen. Nach Enttäuschungen bei Verhandlungen in den Vereinigten Staaten (Key West, 2001) und in Frankreich (Ram- bouillet, 2006) befand jedoch die aserbaidschanische Führung offenbar, dass nur Russland in der Lage sei, Armenien zu einer Änderung ihrer Haltung zu bewegen. Aserbaidschans Säbelrasseln, sein Festhalten an der Stationierung von Scharfschützen und schweren Waffen an der Front sowie seine Offensive im April 2016 zielten darauf $a b$, die internationale Gemeinschaft allgemein - und Russland insbesondere - daran zu erinnern, dass die Situation instabil war und dass $\mathrm{Zu}-$ geständnisse von Armenien aktiv erzwungen werden müssten. Keine dieser Taktiken führte jedoch zu den von Aserbaidschan erhofften Ergebnissen.

\section{Aktivitäten der Minsker Gruppe seit 1992}

Seit ihrer Gründung im Jahr 1992 unternahm die Minsker Gruppe Bemühungen in drei Phasen. Die erste, zwischen 1992 und 1998, war geprägt durch Russlands Bestrebungen, die OSZE sowie die Eindämmungsversuche westlicher Mächte gegenüber Moskau ins Leere laufen zu lassen. Als 1994 ein militärisches Patt erreicht wurde, überging der energische erste russische Verhandlungsführer innerhalb der Minsker Gruppe, Vladimir Kazimirov, die westlichen Unterhändler und startete während eines Treffens der Gemeinschaft Unabhängiger Staaten (GUS) in Bischkek Waffenstillstandsverhandlungen. Sein Ziel war die Entsendung einer russisch geführten Friedenstruppe, bevor der Westen seine zu erwartenden Einwände geltend machen konnte. Aserbaidschan lehnte dies $a b$ und unterzeichnete 
in Baku ein Waffenstillstandsdokument ohne jegliche Mechanismen zur Friedenssicherung.

Im Jahr 1997 traten Frankreich und die Vereinigten Staaten der Minsker Gruppe bei. Wie Russland wurden sie Ko-Vorsitzende der Gruppe, und Kazimirov wurde ersetzt. Gemeinsam entwarfen die Ko-Vorsitzenden einen Friedensplan in zwei Dokumenten, um zunächst vorübergehende Regelungen und dann den finalen Status separat verhandeln zu können (daher die Bezeichnung „Schritt für Schritt“-Plan). Die Präsidenten Armeniens und Aserbaidschans, Ter-Petrosyan und Heidar Alijev, akzeptierten den Plan bei dessen Präsentation im Juli 1997. Keiner von beiden hatte irgendwelche Illusionen, dass ein Übereinkommen bezüglich des Status erzielt werden könne. Beide glaubten, das Abkommen, das sie unterzeichnen sollten, würde Nagornyy Karabach zu einem permanenten De-facto-Protektorat von Armenien machen, im Austausch gegen die Rückgabe besetzter Gebiete an Aserbaidschan. Alijev glaubte, die Abmachung würde das Problem Karabach, das bereits fünf seiner Amtsvorgänger zu Fall gebracht hatte, auf die lange Bank schieben. Die Abmachung kam Ter-Petrosyans Strategie entgegen, eine Abmachung auszuhandeln, bevor Aserbaidschan seinen Ölreichtum ausspielen konnte. Angesichts der internen Opposition blieb Ter-Petrosyan jedoch im März 1997 keine andere Wahl, als den Anführer Nagornyy Karabachs, Robert Kocharyan, als Premierminister zu akzeptieren. Der letzte von Ter-Petrosyans Anhängern, dem noch militärische Kräfte unterstanden, der Verteidigungsminister Vazgen
Sargsyan, verließ ihn wegen des Friedensplans. Im Januar 1998 zwang die Gruppe von Hardlinern, die inzwischen alle Schalthebel der armenischen Streitkräfte kontrollierte, Ter-Petrosyan zum Rücktritt. Kocharyan wurde Präsident, Sargsyan Premierminister, und beide lehnten den Plan der OSZE ab. ${ }^{7}$

Die zweite Phase, 1998 bis 2005, begann mit Verhandlungen zwischen Kocharyan und Alijev, die sie nicht nur vor der jeweiligen Öffentlichkeit, sondern auch vor ihren engeren Mitarbeitenden geheim hielten. Im Jahr 1999 vereinbarten sie mündlich ihren eigenen Plan: einen Landtausch, bei dem Nagornyy Karabach de jure Armenien angegliedert werden würde, im Austausch gegen armenische Gebiete entlang der iranischen Grenze, zwischen Aserbaidschan und seiner Exklave Nachitschewan. Im Herbst 1999 unterrichteten sie die Ko-Vorsitzenden der Minsker Gruppe von dem Plan und baten diese um eine schriftliche Fassung. Nur Wochen später, am 27. Oktober 1999, stürmten bewaffnete Extremisten das armenische Parlament und erschossen eine Anzahl von Regierungsvertretern, darunter den Premierminister Sargsyan und den Parlamentspräsidenten Karen Demirchyan. Daraufhin informierte Kocharyan die Minsker Gruppe, dass er das Friedensabkommen nicht länger unterstützen könne. Die Ko-Vorsitzenden der Minsker Gruppe versuchten, den Plan am Leben zu erhalten, indem sie ihn auf armenische Interessen hin abstimmten - sie behielten den Anschluss von $\mathrm{Na}$ gornyy Karabach an Armenien bei, strichen jedoch die Gebiete, die Aserbaidschan im Austausch dafür erhalten hät- 
te, und ersetzten sie lediglich mit dem Zugang zu einer Straße nach Nachitschewan. Diese Änderung war für Aserbaidschan inakzeptabel; Alijev wies den Plan bei den Verhandlungen in Key West (2001) zurück. Alijev, der sein ganzes Leben damit zugebracht hatte, seine persönliche Machtbasis in Aserbaidschan zu festigen, starb 2003; sein Nachfolger wurde sein Sohn, Ilham Alijev, dessen Machtbasis kaum über seinen Familienverband hinausreichte. Der ältere Alijev konnte zuversichtlich sein, dass er öffentliche Unruhen überstehen würde, selbst wenn er Zugeständnisse machte; der jüngere konnte das nicht. In der Folge waren weder Aserbaidschan noch Armenien fähig zu einem wirklichen Kompromiss.

Während der dritten Phase seit 2005 kehrte man mit einem kurzen Dokument über „Prinzipien“, das schließlich Ende 2007 in Madrid kodifiziert und den Parteien präsentiert wurde, zurück zu einer abgespeckten Version des „Schritt für Schritt"-Plans. Die Madrider Prinzipien, die den Verhandlungsprozess retten sollten, sahen die Rückgabe einiger besetzter Gebiete vor, dazu Garantien für einen Übergangsschutz für Nagornyy Karabach vor militärischen Handlungen seitens Aserbaidschans und schließlich einen nicht näher definierten „bindenden Ausdruck des Volkswillens", der über den letztendlichen Status zu entscheiden haben würde. Ein Übereinkommen über diesen Status würde den Weg frei machen für die Rückgabe der verbleibenden besetzten Gebiete.

Dmitrij Medvedev unternahm während seiner Präsidentschaft in Russland (2008-2012) große Anstrengungen, um zwischen seinen armenischen und aserbaidschanischen Amtskollegen Serzh Sargsyan und Ilham Alijev zu vermitteln. Russland dominierte mittlerweile den Verhandlungsprozess, und zwar mit $\mathrm{Zu}$ stimmung der Vereinigten Staaten und Frankreichs, die nicht so viel politisches Kapital auf höchster Ebene investieren konnten. Nach 1997 war die Minsker Gruppe ein seltenes Beispiel gelungener Kooperation zwischen Russland und dem Westen, und sie bleibt bis heute die Ausnahme, trotz der Spannungen in der Ukraine, in Syrien und anderen Orten. Dieser Erfolg gründet allerdings zum Teil auch auf der Bereitschaft der Vereinigten Staaten und Frankreichs, seit 2008 Russland innerhalb der Gruppe die Initiative zu überlassen.

Sargsyan und Alijev trafen sich weiterhin, Medvedev zuliebe. Jedoch war in Wirklichkeit keiner der beiden daran interessiert, einen Kompromiss auszuhandeln, der innerhalb der eigenen Bevölkerung für Unruhe sorgen würde. Obwohl Russland sich im Vorfeld eines Gipfeltreffens in Kazan 2011 optimistisch gezeigt hatte - man wollte versuchen, die Präsidenten dazu zu überreden, wenigstens ein Abkommen über einige der Prinzipien zu unterzeichnen -, lehnten Alijev und Sargsyan ab. Die „Formel von Kazan“ (die finale, stark verkürzte Wiederholung der Madrider Prinzipien) wird zwar oft zitiert, wurde jedoch gewissermaßen außer Kraft gesetzt, wobei einige einzelne Punkte nach wie vor relevant sind. Die Minsker Gruppe verfiel in einen Ruhezustand, der von der aserbaidschanischen Offensive 2016 über die armenische Revolution von 2018 bis zu 
den Kämpfen im Jahr 2020 anhielt. Während dieser Ereignisse war Russland der wichtigste Vermittler, und sein langfristiges Ziel scheint, wie bereits 1994, noch immer die Entsendung einer russischen Friedenstruppe zu sein.

Somit befinden sich die Beteiligten seit 20 Jahren - seitdem Extremisten die Übereinkunft zwischen Kocharyan und Alijev sabotierten - in „Scheinverhandlungen“, wie es einmal ein russischer Vermittler nannte. Teil dieser Scharade waren die rituellen Schuldzuweisungen an die Minsker Gruppe für die eigene Kompromissunfähigkeit auf allen Seiten. Sie nahm dies hin, wohl wissend, dass man um der Kompromisse wegen, die der Frieden erforderte, diesen Führungspersonen unbedingt politische Deckung geben musste. Indem sie Druck auf die politischen Führungen vermied, deckte die Minsker Gruppe allerdings in der Praxis deren Kurs, Friedensbemühungen zu vermeiden. Vermittler können keinen Frieden schließen; die Krieg führenden Parteien müssen es tun. Die Anführer beider Seiten hatten sich jeweils in die Ecke manövriert: Indem sie versprachen, maximalistische Forderungen ohne Kompromisse zu erfüllen, brachten sie ihre Bevölkerungen dazu zu glauben, dass Kompromiss Verrat bedeute. Die politischen Führer passten sich entsprechend an. Anfang 1993 konnte Heidar Alijev, noch im Exil in seiner Geburtsheimat Nachitschewan, dem amerikanischen Botschafter im Vertrauen sagen: „Selbst als wir Karabach noch hatten, gehörte es uns nicht." Sein Sohn wiederholt heute nur einen einzigen Slogan, „Karabach ist Aserbaidschan“, und verkündet, dass
Aserbaidschan nun nicht mehr wie einst ein „hohes Maß an Unabhängigkeit“ für die Region anbiete. ${ }^{8}$

Die Bemühungen der OSZE haben noch einen weiteren Aspekt: Im Jahr 1996 ernannte das amtierende Vorsitzland einen persönlichen Repräsentanten, der in der Region anwesend sein sollte, im Gegensatz zu den Ko-Vorsitzenden, die jeweils von Zeit zu Zeit einflogen. Seit 24 Jahren erfüllt Botschafter Andrzej Kasprzyk diese Rolle. In Ermangelung einer Friedenstruppe oder einer dauerhaften Beobachtungsmission führte Kasprzyks Büro die wenigen Überwachungstätigkeiten durch, die überhaupt stattfinden konnten, und leitete kurze Beobachtungsmissionen in einigen wenigen $\mathrm{Ge}$ bieten auf beiden Seiten der Kontaktlinie. Da diese jedoch im Voraus von beiden Seiten bewilligt werden mussten, waren ihre Erkenntnisse von eingeschränktem Nutzen.

\section{Politische Auswirkungen der letzten Kampfhandlungen}

Die Offensive, die Aserbaidschan im September 2020 begann, war eine Fortsetzung der beiden politischen Strategien, mit denen das Land seit Jahren versuchte, die Tatsachen vor Ort zu verändern: Gebiete zurückgewinnen und gleichzeitig den internationalen Druck auf Armenien konzentrieren. Indem es besetzte Gebiete zurückgewann, konnte Aserbaidschan Armenien seine Verhandlungsmasse für künftige Gespräche entziehen. Durch die militärische Unterstützung der Türkei hatte Aserbaidschan größeren Erfolg als 
erwartet. Nachdem sie rasch durch vier Provinzen vorgerückt war, eroberte die aserbaidschanische Armee am 7. November die Bergfestung von Şuşa im Herzen Nagornyy Karabachs zurück, ermöglichte damit den Artilleriebeschuss der Hauptstadt Stepanakert und sperrte den LachinKorridor mit der Straße, die Stepanakert mit Armenien verbindet. ${ }^{9}$

Der zweite Hebel in der Strategie Aserbaidschans bestand darin, internationalen Druck aufzubauen. Durch die Androhung eines breiteren Krieges und gröBerer Instabilität nahe den russischen Grenzen hoffte die aserbaidschanische Führung, Russland dazu zu bringen, nachhaltigen Druck auf Armenien auszuüben. Durch den Erfolg der Militärkampagne gelang dies. Beide Strategien wurden durch die Unterstützung der Türkei ermöglicht. Istanbul leistete nicht nur militärische Hilfe, einschließlich der letztlich kampfentscheidenden Bayraktat-TB2-Kampfdrohnen, sondern auch diplomatische Unterstützung, um Alijevs Druck auf Russland zu verstärken. ${ }^{10}$ Die Türkei transportierte Söldner von Syrien nach Aserbaidschan und stationierte F-16-Kampfflugzeuge in Ganja; in militärischer Hinsicht hatten diese wenig Bedeutung, aber sie signalisierten Russland unmissverständlich, was passieren könnte, sollte die Situation außer Kontrolle geraten. ${ }^{11}$

Russlands Entgegnung, zusammengefasst in einer Aussage des Außenministers Lavrov vom 14 . Oktober, ${ }^{12}$ war lediglich ein Angebot, die Situation zwischen den Konfliktparteien einzufrieren, mit dem Versprechen, fünf Provinzen zurückzugeben, in denen Aserbaidschan bereits schnell vorrückte und die es ohnehin beinahe erobert hatte, während das Schicksal von Lachin, Kelbajar und Şuşa einer endgültigen politischen Lösung überlassen wurde. Lavrov machte deutlich, dass der Preis hierfür die Entsendung russischer Friedenstruppen war.

Aserbaidschan hatte seit mittlerweile 25 Jahren russische Friedenstruppen abgelehnt. Die Entsendung eines Kontingents russischer Friedenstruppen würde das Regime in Nagornyy Karabach an der Macht erhalten und den Konflikt für eine weitere Generation einfrieren. Der Vorteil, den Aserbaidschan durch die volle Unterstützung durch die Türkei gewann, wäre hinfällig geworden, genauso wie das moderne Waffenarsenal, das den Erfolg gebracht hatte. Alijev würdigte Lavrovs Angebot nicht einmal einer Antwort. Die Verhandlungen, die nacheinander von Russland, Frankreich und den USA betreut wurden, führten zu „humanitären“ Waffenstillständen, die manchmal innerhalb von Minuten zusammenbrachen, während Aserbaidschan seine Offensive vorantrieb. Alijev wischte internationale Bedenken beiseite, indem er behauptete, er setze lediglich die Resolutionen des UN-Sicherheitsrats um, die 1993 verabschiedet worden waren, als Armenien mit seinen Offensiven große Teile Aserbaidschans eingenommen und die Bevölkerungen vertrieben hatte. ${ }^{13}$

Armenien und Nagornyy Karabach, die über Jahre hinweg den Ausbau der militärischen Fähigkeiten Aserbaidschans ignoriert hatten, fanden keine wirkungsvolle Antwort auf den schnellen Vormarsch Aserbaidschans. Premierminister Pashinyan appellierte an Putin um Hilfe, 
erhielt aber am 31. Oktober eine kühle Antwort, nicht von Putin selbst, sondern in einer Erklärung des russischen Ministeriums für Auswärtige Angelegenheiten, welche die übliche Antwort wiederholte, dass Russland im Falle eines direkten Angriffs auf Armenien seinen zugesicherten Verpflichtungen gemäß dem Vertrag über kollektive Sicherheit nachkommen werde. Inzwischen forderte Russland alle Beteiligten des Konflikts dazu auf, den Waffenstillstand einzuhalten, den sie am 10. Oktober in Moskau vereinbart hatten. ${ }^{14}$ Damit wurde Russland wieder einmal mit dem wiederkehrenden Dilemma im Südkaukasus konfrontiert: Es wollte ein Gleichgewicht zwischen Armenien und Aserbaidschan finden, seinen Einfluss auf beide Staaten erhalten und gleichzeitig eine Destabilisierung durch eine der beiden Parteien minimieren.

Russland erscheint nicht gewillt, Pashinyan zu unterstützen, der in den russischen Medien als Anführer einer „Farbenrevolution“ unbeliebt ist. Als er an die Macht kam, versuchte Pashinyan, Putin zu versichern, dass er „Demokratie als einen festen Glauben betrachte, und nicht als geopolitische Orientierung “. 15 Obwohl diese Differenzierung Putin möglicherweise nicht beschwichtigte, scheint es schwer vorstellbar, dass das Missfallen eines einzigen Anführers die seit Generationen bestehende russisch-armenische Allianz beenden könnte. Wahrscheinlicher ist, dass Putin einen weitaus größeren Erfolg in Reichweite glaubte: durch Zugeständnisse an Alijev bei einigen seiner Kriegsziele eine strategische Partnerschaft mit Aserbaidschan zu schmieden. Wenn dadurch Nagornyy
Karabachs Hoffnungen auf eine Vereinigung mit Armenien oder auf Unabhängigkeit zerschlagen werden könnten, käme Armenien in eine Zwickmühle. Seine Sicherheit wäre weiterhin abhängig von Russland, wie das militärische Abenteuer der Türkei in Aserbaidschan gerade im rechten Moment demonstrierte. Putin hatte bereits einen Präzedenzfall geschaffen: Im Jahr 2003 war er bereit, seine Klienten in Transnistrien zu opfern, als Moldawien Russland im Rahmen des KozakMemorandums das Recht auf Truppenstationierung und geopolitische Zusammenarbeit anbot.

Das Engagement der Türkei im Krieg um Karabach stellte einen scharfen Bruch mit der Politik in der Vergangenheit dar: Der frühere Präsident Süleyman Demirel pflegte zu sagen, es würde nur Minuten dauern, nach Karabach hineingezogen zu werden, aber Jahre, um wieder herauszukommen. Trotz Spekulationen über Präsident Recep Tayyip Erdoğans "neo-osmanische" Strategie finden seine scheinbar opportunistischen Interventionen immer dann statt, wenn die Aussicht winkt, den Fußabdruck und den Einfluss der Türkei zu vergrößern. In der türkischen Öffentlichkeit gibt es breite Unterstützung für Aserbaidschan. Armenische Erklärungen im August 2020, die auf eine Wiederbelebung des Vertrags von Sèvres (1920) abzielten, haben Erdoğan wohl dazu veranlasst, zur Tat zu schreiten. ${ }^{16}$ Für die Türkei stand ansonsten wenig auf dem Spiel, hatte sie doch bereits direkte Straßen- und Schienenverbindungen nach Armenien, Aserbaidschan und Zentralasien durch Georgien. Die jährlichen Exporte der Türkei nach Armenien in Höhe 
von 248 Millionen US-Dollar werden sich vermutlich nicht bemerkenswert erhöhen - und ihr Wegfall würde kaum ins Gewicht fallen. ${ }^{17}$

Frankreich und die Vereinigten Staaten, die westlichen Ko-Vorsitzenden der Minsker Gruppe, welche die Initiative an Russland abgetreten hatten, versuchten, Waffenstillstände zu vermitteln, taten aber wenig, wenn diese zusammenbrachen. Die Passivität der westlichen Mächte gab sowohl Russland als auch der Türkei mehr Handlungsspielraum.

\section{Der Waffenstillstand und die Folgezeit}

Seit Jahrzehnten hatte sich Aserbaidschan den russischen Forderungen nach einer Friedenstruppe widersetzt. Warum also akzeptierte Alijev am 9. November den russischen Waffenstillstandsplan, der die Entsendung von 1.960 schwer bewaffneten Friedenstruppen vorsah? ${ }^{18}$ Vom militärischen Standpunkt aus betrachtet hätte die aserbaidschanische Armee weiter vorstoßen und sowohl Lachin einnehmen können - wodurch Nagornyy Karabach von Armenien abgeschnitten worden wäre - als auch die Hauptstadt Stepanakert. Die Aussicht, weitere 80.000 Armenier*innen zu Flüchtlingen zu machen, kann Alijev nicht groß abgeschreckt haben, dessen Regierung immer noch 850.000 aserbaidschanische Flüchtlinge und Binnenvertriebene der Kämpfe in den frühen 1990er-Jahren betreuen muss. ${ }^{19}$

Um diese Frage zu beantworten, sollte man zunächst untersuchen, was die Friedenstruppen leisten können. Zum einen verkörpern sie eine (mindestens) fünfjährige Sicherheitsgarantie für Nagornyy Karabach, unabhängig davon, ob eine Einigung über den Status erzielt wird oder nicht. Zweitens projizieren sie russische Macht in den ganzen Südkaukasus und erfüllen somit ein lang gehegtes Ziel der russischen Regierung. Drittens werden russische Friedenstruppen das Transportwesen zwischen Nagornyy Karabach und Armenien überwachen. Russische Grenzwächter werden den Verkehr zwischen der aserbaidschanischen Exklave Nachitschewan und dem Rest von Aserbaidschan durch die armenische MeghriRegion kontrollieren; sie könnten an der Schienenbrücke über den Fluss Aras bei Julfa in Nachitschewan auch die Zugverbindungen zwischen Armenien und dem Iran kontrollieren. Diese Aufgaben werden den russischen Einfluss auf den Handel und die Wirtschaft in der Region erhöhen.

Warum also stoppte Alijev seine Offensive und stimmte dieser Ausweitung russischer Macht zu? Es ist anzunehmen, dass Putin und möglicherweise auch Erdoğan genügend Druck auf ihn ausübten. Möglicherweise hatte Putin auch bereits ein Übereinkommen mit Erdoğan getroffen. Es ist wahrscheinlich, dass $\mathrm{Pu}$ tin Alijev Versprechen machte, um seine Zustimmung zu gewinnen. Die Stabilität des Waffenstillstands wird davon abhängen, wie sehr Russland diese geheimen Versprechen und Übereinkünfte einhalten wird, aber es liegt in der Natur der Sache, dass wir diese Frage unmöglich beantworten können. Die Schlussfolgerung liegt nahe, dass bezüglich der Rolle der Türkei Unstimmigkeit herrscht. Alijev 
erwartete offensichtlich, dass die Rolle der Türkei derjenigen Russlands gleichgestellt wäre; Russland stellte sich vor, dass die Türkei kleinere technische Hilfestellungen leisten würde. ${ }^{20}$ Russland und die Türkei arbeiten noch daran, ihre wie auch immer gearteten Übereinkünfte auszuarbeiten. Russland hat bisher seinen Willen durchgesetzt, aber falls Erdoğan den Eindruck bekommt, dass ihm eine angemessene Rolle vorenthalten wird, wird er vermutlich nicht passiv bleiben.

Lavrov hat deutlich gemacht, dass Russland die Zustimmung des UN-Sicherheitsrats suchen wird, was zumindest eine Zustimmung zu einer russischen Friedenstruppe impliziert und Veränderungen in ihrer Zusammensetzung verhindern würde. ${ }^{21}$ Seit der Medvedev-Initiative bis 2016, so auch in der „Kazaner Formel", schien Russland das Prinzip einer multinationalen, neutralen Friedenstruppe unter OSZE-Aufsicht akzeptiert zu haben, wozu auch Obergrenzen für Truppen- und Führungskontingente gehören sollten, um zu verhindern, dass eines der Länder die Rolle der Friedenstruppen zum eigenen Vorteil monopolisieren konnte. Nach der aserbaidschanischen Offensive im April 2016 versuchte Lavrov jedoch, Aserbaidschan und Armenien dazu zu drängen, eine russische Friedenstruppe zu akzeptieren. Bezeichnend für Russlands Prioritäten ist die Tatsache, dass die am detailliertesten ausformulierten Klauseln des heutigen Waffenstillstandsplans diejenigen sind, die die russische bewaffnete Präsenz in der Region regeln und keine friedenserhaltende Rolle für die OSZE vorsehen. Einen potenziellen Effekt könnte die größere Rolle
Russlands, durch die der neue Frontverlauf eingefroren wird, allerdings haben: Während die Minsker Gruppe bislang für die politischen Führer der verfeindeten Lager oft als Sündenbock für das Scheitern der Friedensbemühungen herhalten musste, könnten nun (insbesondere in Aserbaidschan) stattdessen Russland und seine Friedenstruppen diese Rolle übernehmen.

\section{Verhandlungsaussichten}

Das Waffenstillstandsabkommen enthält keine Hinweise auf zukünftige Verhandlungen über den Status von Nagornyy Karabach, wie Alijev in seiner Ansprache an die Nation am 10. November triumphierend feststellte. ${ }^{22}$ Ein verkleinertes Nagornyy Karabach ohne Aussicht auf Änderung und ohne jeden Status zu belassen, hieße jedoch letztlich, zukünftige Probleme in Kauf zu nehmen. Aserbaidschan mag noch nicht bereit sein, die Einwohner*innen von Karabach wieder „mit einem hohen Grad an Autonomie“ aufzunehmen, und Letztere sind womöglich zu verbittert, um etwas mit Aserbaidschan zu tun haben zu wollen. Letztendlich wird jedoch die Wiedereröffnung der Transport- und Handelswege praktische Fragen aufwerfen, die auf dem Verhandlungsweg gelöst werden müssen. Aserbaidschan könnte argumentieren, dass diese Frage nun, nach Beendigung des Krieges mit Armenien, eine innenpolitische Angelegenheit Aserbaidschans sei (mit dem Präzedenzfall der Beendigung des Mandats der OSZE-Hilfsgruppe in Tschetschenien durch Russland, nach- 
dem es auf eine militärische Lösung dieses Krieges gedrängt hatte). Russland und andere könnten allerdings auch Druck auf Aserbaidschan ausüben, Verhandlungen unter internationaler Vermittlung aufzunehmen.

Für den Fall, dass solche Verhandlungen stattfinden, hat Russland bereits klargemacht, dass es keine Veränderungen am Verhandlungsformat der Minsker Gruppe zulassen wird. ${ }^{23}$ Wie oben erwähnt, dominiert Russland dieses Format seit über einem Jahrzehnt. Russland hat die klare Absicht, diese Vormachtstellung durch die Beibehaltung des Formats zu behaupten. Dieser Vorschlag ist den am häufigsten genannten Alternativen vorzuziehen, wie zum Beispiel, die gegenwärtigen Ko-Vorsitzenden durch andere, nicht näher genannte Länder zu ersetzen, den Armenier*innen aus Karabach einen eigenen Platz am Verhandlungstisch zuzuweisen oder die Türkei zu einem KoVorsitzenden zu machen. Alle diese Vorschläge würden die Verhandlungen behindern:

- Neue Ko-Vorsitzende müssten schnell viel dazulernen. Die Ko-Vorsitzenden sollten engere Beziehungen zu den anderen Staaten der Minsker Gruppe pflegen, beispielsweise mit der Türkei und Schweden, das im Jahr 2021 den OSZE-Vorsitz innehaben wird. Engere Beziehungen könnten die Transparenz erhöhen, doch eine Machtverschiebung innerhalb der Gruppe ist unwahrscheinlich.

- Die Armenier*innen in Karabach, deren Anführer von 1998 bis 2018 in Armenien regierten, waren während die- ser Zeit bei den Verhandlungen gut vertreten. Armenien hat wiederholt gefordert, Nagornyy Karabach müsse als eigene Partei an den Verhandlungen beteiligt werden - nicht, um eine Lösung zu erreichen, sondern um stärkere Argumente für die internationale Anerkennung zu haben und um Armenien nicht in die Situation zu bringen, Kompromisse zurückweisen zu müssen. Bei neuen Verhandlungen könnten die Einwohner*innen von Karabach entweder Armenien ersetzen (falls die Konfliktparteien akzeptieren, dass es sich um einen internen Disput von internationaler Tragweite handelt) oder sich der armenischen Delegation anschließen (um zu zeigen, dass es sich weiterhin um einen internationalen Konflikt handelt).

- Eine Einbindung der Türkei als KoVorsitzender würde die Atmosphäre vergiften und gäbe den irredentistischen Hardlinern unter der armenischen Diaspora im Westen - deren Vorfahren dem Genozid durch das Osmanische Reich zum Opfer fielen ein moralisches Veto bezüglich der armenischen Verhandlungspositionen.

Von verschiedener Seite wurde der Vorschlag eingebracht, die Verhandlungen an die Vereinten Nationen zu übergeben, um dem Prozess neuen Schwung $\mathrm{zu}$ verleihen. Einige Stolpersteine müssen noch überwunden werden, so etwa der Widerstand einiger Interessenvertreter und die Existenz der vier UN-Sicherheitsrats-Resolutionen, die Aserbaidschan zur Rechtfertigung seines militärischen Eingreifens hinzuzieht. ${ }^{24}$ Russland wür- 
de in seinen Bemühungen um eine weitere UN-Sicherheitsrats-Resolution gestärkt, welche seine Verlegung von Friedenstruppen rechtfertigen und die russische Präsenz vor Ort nach internationalem Recht zementieren würde. Nichts weist darauf hin, dass die Vereinten $\mathrm{Na}-$ tionen mehr Erfolg dabei hätten, die politischen Führungen von Armenien und Aserbaidschan zu überzeugen als die KoVorsitzenden der Minsker Gruppe, deren Staaten ausnahmslos ständige Mitglieder des Sicherheitsrats sind.

Es wurden auch Forderungen laut, die Minsker Konferenz (mit Beteiligung aller Mitglieder der Minsker Gruppe) einzuberufen, die ursprünglich für 1992 vorgesehen war, die jedoch nach etlichen Einwänden von beiden Seiten blockiert wurde. Die Unfähigkeit, die Konferenz einzuberufen, war die Ursache für die gegenwärtige Konstellation mit den Ko-Vorsitzenden. Allgemein wird angenommen, dass die Einberufung der Konferenz nur dann Sinn machen würde, wenn dort ein Text verabschiedet würde, an dem die Beteiligten bereits bedeutenden Fortschritt erreicht hätten, und wenn sie auch Anlass für eine Geberkonferenz zur Stärkung der Stabilität in der Folge des Konflikte sein könnte. Würde die Konferenz zu früh einberufen, wäre eine Fortsetzung der unproduktiven gegenseitigen Schuldzuweisungen zu erwarten, die bereits im Ständigen Rat der OSZE zu beobachten sind. Ein weiterer seit Jahren vorgebrachter Vorschlag ist eine Anerkennung der Unabhängigkeit von Nagornyy Karabach durch Armenien. Dieser Schritt könnte die Moral auf der armenischen Seite schwächen, doch davon abgesehen wäre es eine Geste ohne Konsequenzen. ${ }^{25}$

\section{Die Zukunft der Minsker Gruppe}

Zum Schluss zeichnet sich eine mögliche Rolle für die Minsker Gruppe der OSZE in dem Waffenstillstand $a b$, der durch Russland ausgehandelt wurde, und zwar in den Verhandlungen über vertrauens- und sicherheitsbildende Maßnahmen (VSBM) zwischen den Konfliktparteien, über den zukünftigen Status von Nagornyy Karabach, und über die Bestandteile eines regionalen Friedensabkommens.

Am Anfang der VSBM muss die Aufgabe stehen, die Region für die gegenwärtigen Einwohner*innen sicher zu machen und die Rückkehr der Vertriebenen zu ermöglichen. Weder die USA noch die EU werden voraussichtlich große Truppenkontingente vor Ort stationieren oder Russland, Armenien oder Aserbaidschan Geldmittel ohne Gegenprüfung zur Verfügung stellen. Die OSZE sollte eine Präsenz vor Ort etablieren, um die Projekte auszuhandeln und zu realisieren, die sowohl für die verbleibende als auch für die zurückkehrende Bevölkerung unerlässlich sind. Eine solche Präsenz könnte als Nachfolge oder als Ersatz für das Büro des persönlichen Repräsentanten des Generalsekretärs gestaltet werden. Zu den Aufgaben gehören neben allgemeiner humanitärer Hilfe für den Wiederaufbau von Wohnhäusern und anderen wichtigen Infrastrukturen die folgenden Elemente: 
- Minenräumen

- Abzug oder Entflechtung von Truppen und schweren Waffen

- Polizei und Polizeiausbildung

- eine zukünftige Rolle der OSZE bei der militärischen und/oder zivilen Beobachtung des Waffenstillstands

- Einrichtung einer Kommunikationsinfrastruktur für zivile Kontakte zwischen den beiden Seiten, inklusive Zugang zu nachhaltiger Transportinfrastruktur zwischen Armenien und Nagornyy Karabach

- Dialog im Rahmen des Hohen Flüchtlingskommissars der Vereinten Nationen und des Internationalen Komitees vom Roten Kreuz, um die humanitären Bedürfnisse der verbleibenden Karabach-Armenier*innen sowie der zurückkehrenden aserbaidschanischen Binnenflüchtlinge festzustellen

- Schutz und Wiederherstellung des religiösen und kulturellen Erbes in der Region auf beiden Seiten ${ }^{26}$

- Einrichtung von Märkten auf beiden Seiten mit dem Ziel, später von beiden Seiten zugängliche Märkte zu schaffen

- Einrichtung von gemeinsamen Arbeitsgruppen, um die notwendige $\mathrm{Zu}$ sammenarbeit in den Bereichen Infrastruktur, Gesundheitswesen und Wirtschaft (z.B. Banken) zu gewährleisten, sowie Bemühungen um eine Beschränkung der feindseligen Rhetorik, etwa durch Einbezug der Zivilgesellschaft. Auch wenn die Erfolgsaussichten anfangs dürftig wirken mögen, leistet jeder noch so kleine Schritt einen Beitrag. Solche Bemühungen könnten irgendwann auf gemeinsa- me zivilgesellschaftliche Projekte ausgeweitet werden, zum Beispiel für Nachwuchsführungskräfte.

Verhandlungen über die Zukunft von Nagornyy Karabach und seiner Einwohner"innen werden langwierig und schwierig sein. Der Waffenstillstand hat neue Kontaktlinien in Stein gemeißelt. Beide Seiten haben soeben wieder erlebt, dass man mit Waffengewalt die Fakten vor Ort ändern kann, und werden daher eine Weile brauchen, um sich wieder mit dem neuen Gleichgewicht abzufinden. Dies bedeutet jedoch keine Verschnaufpause für die Westmächte in der Minsker Gruppe - wozu neben den USA und Frankreich auch Deutschland, die Türkei und Schweden gehören. Diese müssen zusammenarbeiten, um eine $\mathrm{Al}$ ternative zum russischen Plan zu entwickeln, da dieser unweigerlich Klauseln enthalten wird, die den russischen Fußabdruck in der Region zementieren. In diesem Zusammenhang besteht die wichtigste Vorbereitung darin, die Bedürfnisse der betroffenen Bevölkerungsgruppen in der Region zur Kenntnis zu nehmen. Entscheidungsträger*innen aller externen Mächte (inklusive Türkei und Russland) müssen mehr über die Geschichte, die Kultur und die Menschen dieser Länder lernen.

Für eine Normalisierung der Beziehungen ist ein regionales Friedensabkommen erforderlich, und zwar in erster Linie eines zwischen Armenien und Aserbaidschan; und die internationalen Grenzen müssten geöffnet werden, zum Beispiel zwischen der Türkei und Armenien. Mit der Unterzeichnung des Alma-Ata- 
Protokolls vom 21. Dezember 1991, das zur Gründung der GUS führte, verpflichteten sich Armenien und Aserbaidschan zusammen mit anderen ehemaligen Sowjetrepubliken zur Anerkennung ihrer gegenseitigen Unabhängigkeit innerhalb der früheren Grenzen der Sowjetunion. Im Karabach-Konflikt waren jedoch zu diesem Zeitpunkt die Kampfhandlungen bereits voll ausgebrochen, und die beiden Staaten einigten sich niemals auf normale Beziehungen oder anerkannte Grenzziehungen. Aserbaidschan betrachtete den Konflikt um Karabach als Aggression Armeniens, und Armenien betrachtete ihn als Aggression Aserbaidschans gegenüber der Bevölkerung von Nagornyy Karabach. Diese Faktoren wurden nach den Interventionen Russlands auf der Krim und in der Ostukraine weiter verkompliziert, da diese Auswirkungen auf die Protokolle von Alma-Ata hatten.

In einem ersten Schritt sollte die Minsker Gruppe versuchen, einen formellen Waffenstillstand entlang der Grenze zwischen Aserbaidschan und Armenien auszuhandeln. Dies ist gerade mit Blick auf die voraussichtliche Rückkehr der aserbaidschanischen Binnenflüchtlinge in ihre ehemaligen Wohnorte in den Provinzen Kelbajar und Lachin von besonderer Bedeutung. Entlang dieser Grenze hat es immer wieder $\mathrm{Zu}$ sammenstöße gegeben, zuletzt im Juli 2020. Ein Waffenstillstand könnte hier Chancen für eine weitere Verbesserung der Beziehungen schaffen.

Ein regionales Abkommen könnte auch darauf abzielen, die Landgrenze zwischen Armenien und der Türkei ganz zu öffnen. Seit der Sowjetära gab es eine wöchentliche Zugverbindung zwischen Kars in der Türkei und Leninakan (jetzt: Gyumri) auf der armenischen Seite. Im Jahr 1993 merkte die Türkei Gelder vor, die für die Öffnung der Landgrenze für Fahrzeuge und Lastwagen vorgesehen waren. Im März 1993 jedoch entschied sich die Türkei angesichts der Offensive gegen Kelbajar, die teilweise von Armenien aus vorbereitet worden war, den Zugverkehr einzustellen und ihre Pläne für die Straße entlang der Grenze aufzugeben. Weitere Normalisierungsversuche im Jahr 2009 mit Unterstützung der USA stießen auf den Widerstand wichtiger Interessengruppen. Als Kelbajar wieder unter aserbaidschanischer Kontrolle war, verkehrten sich die Ereignisse, die zur Grenzschließung geführt hatten, in ihr Gegenteil. Eine Wiederherstellung der grenzüberschreitenden Kommunikation könnte eine Gelegenheit bieten, die generelle Normalisierung voranzutreiben.

\section{Fazit}

Die neuesten Kriegshandlungen widersprechen der psychologischen Ausgangssituation der vergangenen 26 Jahre: Zum ersten Mal seit dem Waffenstillstand von 1994 erkennen sowohl Baku als auch Eriwan nun, dass man mit militärischer Gewalt tatsächlich politische Ziele erreichen kann. Beide Lager hatten sich an das militärische Patt gewöhnt und passten ihre Strategien entsprechend an. Militärische Operationen dienten nur der Anschauung statt irgendwelchen strategischen Zielen. Sie waren für ein innenpolitisches oder internationales Publikum 
bestimmt, sollten jedoch keine materielle Veränderung vor Ort bewirken. Diese Haltung ist nun unwiderruflich vorbei. Russische Friedenstruppen mögen verhindern können, dass es zu weiteren Gewaltausbrüchen kommt, doch sie können die Erkenntnis auf beiden Seiten nicht rückgängig machen, dass eine Wiederaufnahme der Kämpfe das Gleichgewicht in der Zukunft wieder verschieben könnte.

Es ist daher unerlässlich, dass multilaterale Verhandlungen wieder aufgenommen werden. Verhandlungen, die einseitig - zum Beispiel durch Russland oder aufgrund geheimer Absprachen zum Beispiel mit der Türkei - durchgeführt werden, dienen weder den Interessen der beiden Konfliktparteien, noch helfen sie der jeweiligen Bevölkerung. Es ist die Aufgabe der Minsker Gruppe - das heißt, aller Ko-Vorsitzenden mit Unterstützung aller restlichen Mitgliedsstaaten - ihrer Verantwortung gerecht zu werden, Vertrauen und Sicherheit zwischen den Kriegsparteien aufzubauen und gemeinsam neue Pläne zu formulieren und auszuhandeln, um einen gerechten und anhaltenden Frieden für die ganze Region zu schaffen.

\section{Notizen}

Die Autoren bedanken sich bei den Mitgliedern der Peer-Review-Gruppe, den Kolleg*innen in den angeschlossenen Instituten und anderen Kolleg*innen, mit denen wir unsere Beitragsentwürfe vertrauensvoll geteilt haben und die uns in jeder Phase mit wertvollen Einsichten unterstützt haben.
Ein Hinweis zur Terminologie: In Anlehnung an de Waal bezeichnet „Nagornyy Karabach" das politische Gebiet, das zu Sowjetzeiten „Autonomes Gebiet Bergkarabach“ hieß („Nagorno-Karabakhskaya Avtonomnaya Oblast“, NKAO), heute in Armenien als Artsakh bekannt (Thomas de Waal, Black Garden, New York und London: New York University Press, 2003). „Armenien“ bezeichnet die Republik Armenien. „Karabach-Konflikt" bezeichnet den Konflikt, der 1988 ausbrach, als der Oberste Sowjet der NKAO die sowjetischen Behörden mit einer Petition zum Transfer der NKAO von der Sowjetrepublik Aserbaidschan an die Sowjetrepublik Armenien aufforderte. Aus Platzgründen wurden armenische Ortsnamen transliteriert; bei Ortsnamen in Azeri wurden der Verständlichkeit halber nur die nicht-lateinischen Buchstaben ersetzt. In den Fußnoten verweist "(ÜB) “ darauf, dass ein Titel übersetzt wurde.

3 UN-Sicherheitsrats-Resolutionen 822 (30. April 1993), 853 (29. Juli 1993), 874 (14. Oktober 1993) und 884 (12. November 1993).

4 Jirair Libaridian, ,Why Negotiations Failed“, Armenian Mirror-Spectator, 2 November 2020, https://mirrorspectator.co m/2020/11/02/why-negotiations-failed/.

5 Siehe z.B., Philip Remler, Chained to the Caucasus, IPI, 2016, https://www.ipinst.o rg/wp-content/uploads/2016/05/1605-Cha ined-to-the-Caucasus.pdf.

6 „PM addresses the nation“, Office of the Prime Minister of the Republic of Armenia, 12 November 2020, https://www.pri meminister.am/en/statements-and-messa ges/item/2020/11/12/Nikol-Pashinyan-Sp eech/.

„The step-by-step approach [...] cannot serve as a basis for resolution." Robert Kocharyan, in einem undatierten Brief an die Ko-Vorsitzenden der Minsker Gruppe, Anfang 1998. 
„Alijev schließt jeglichen Sonderstatus für Nagornyy Karabakh aus“ (ÜB), TASS, 17. November 2020, siehe: https:/tass.ru/ mezhdunarodnaya-panorama/10023751? noredir=true.

Ilham Aliyev, Facebook, November 2020, https:/www.facebook.com/PresidentIlha mAliyev/photos/a.10151996474470315/1 0164122626875315/.

10 Zum Thema Drohnen: „Seit dem Karabakh-Konflikt sprechen alle von der ,Drohnen-Revolution'“ (ÜB), Meduza, 19. November 2020, siehe: https://meduz a.io/feature/2020/11/19/posle-karabahsko go-konflikta-vse-govoryat-o-revolyutsii-dr onov. Zur diplomatischen Unterstützung: „Erdoğan: Es ist an der Zeit, Nagornyy Karabakh zu lösen" (ÜB), Deutsche Welle Türkçe, 29. September 2020, siehe: https://www.dw.com/tr/erdoğan-da ğlk-karabağda-çözüm-vakti-geldi/a-55079 318.

11 Kareem Fahim/Isabelle Khurshudyan/Zakaria Zakaria, „Deaths of Syrian mercenaries show how Turkey, Russia could get sucked into Nagorno-Karabakh conflict", The Washington Post, 14 October 2020, https://www.washingtonpost.c om/world/middle_east/azerbaijan-armen ia-turkey-nagorno-karabakh/2020/10/13/2 cdca1e6-08bf-11eb-8719-0df159d14794_st ory.html.

12 "Lavrov verlangte russische Friedenstruppen in Nagornyy Karabakh” (ÜB), Regnum.ru, 14. Oktober 2020, https://regnu m.ru/news/polit/3089582.html; „Interview von Außenminister Lavrov mit russischen und ausländischen Medien zu laufenden internationalen Angelegenheiten, Moskau, 12. November 2020", Ministerium für Auswärtige Angelegenheiten, 14. Oktober 2020, https://www.mid. $\mathrm{ru} / \mathrm{ru} /$ press_service/minister_speeches/-/as set_publisher/7OvQR5KJWVmR/content /id/4381977.

13 Ilham Aliyev, Facebook, 31. Oktober 2020, https://www.facebook.com/Preside
ntIlhamAliyev/photos/a.10151996474470 315/10164085858605315/.

14 „Erklärung des russischen Ministers für Auswärtige Angelegenheiten zum Appell des Premierministers der Republik Armenien..." (ÜB), Außenministerium der Russischen Föderation, 31. Oktober 2020, https:/www.mid.ru/ru/press_servic e/spokesman/official_statement/-/asset_p ublisher/t2GCdmD8R $\bar{R}$ Ir/content/id/441 1939.

15 Mikayel Zolyan, „Can 'New Armenia' disentangle democratic change from geopolitics?" , OC Media, 25. Juli 2018, https: //oc-media.org/features/analysis-can-newarmenia-disentangle-democratic-change-f rom-geopolitics/.

16 Jirair Libaridian, „A step, this time a big step, backwards", Aravot, 1 September 2020, https://www.aravot-en.am/2020/09 /01/263436/?fbclid=IwAR0Fxh9xluqFMX cvcLu3JNqtuERdX4Oxca5B5o7EjUrp94t PBABIStLUbyg. Mit dem Vertrag von Sèvres wollten die Siegermächte des Ersten Weltkriegs das Osmanische Reich aufteilen. Der Vertrag wurde nie ratifiziert und wurde aufgrund weiterer Verträge, die von verschiedenen Sèvres-Parteien wie Armenien (Vertrag von Kars, 1921) unterzeichnet wurden, irrelevant. Er wurde durch den Vertrag von Lausanne zwischen der Türkei und den Alliierten ersetzt. Dieser wurde zu einem Gründungsdokument der Türkischen Republik.

17 „Armenia“, Observation of Economic Complexity, https://oec.world/en/profil e/country/arm.

18 „Erklärung des Präsidenten der Republik Aserbaidschan, des Premierministers der Republik Armenien und des Präsidenten der Russischen Föderation" (ÜB). Büro des russischen Präsidenten, 10. November 2020, http://kremlin.ru/events/presid ent/news/64384.

19 UNHCR, Azerbaijan: Analysis of gaps in the protection of internally displaced persons (IDPs), October 2009, p. 3, https://w ww.unhcr.org/4bd7edbd9.pdf. 
20 Ilham Aliyev, „Ilham Aliyev und der russische Präsident Vladimir Putin trafen sich per Videokonferenz " (ÜB), Facebook, 10. November 2020, https://www.f acebook.com/PresidentIlhamAliyev/vide os/831550284329696; Lavrov, 12. November 2020, in der oben (Anm. 12) genannten Mitteilung.

21 Lavrov, Pressekonferenz am 12. November 2020, oben zitiert (Anm. 12).

22 Ilham Aliyev, „Ilham Aliyevs Rede an die Nation" (ÜB), Facebook, 10. November 2020, https://www.facebook.com/Preside ntIlhamAliyev/videos/402316387790506.

23 Lavrov, Pressekonferenz am 12. November 2020, oben zitiert (Anm. 12).

24 Ilham Aliyev, Facebook, 31. Oktober 2020, https://www.facebook.com/Preside ntIlhamAliyev/photos/a.10151996474470 315/10164085858605315/.

25 An einer Pressekonferenz am 17. November 2020 tadelte der russische Präsident Putin Armenien, weil es dem russischen Beispiel im Falle der Krim nicht gefolgt war. Demnach hätte es die Unabhängigkeit Nagornyy Karabachs anerkennen, eine Vereinigung herbeiführen und somit die internationale Gemeinschaft vor vollendete Tatsachen stellen sollen. Jedoch hat ein Großteil der internationalen Gemeinschaft die Handlungen Russlands als Verstöße gegen internationales Recht gebrandmarkt und schmerzhafte Sanktionen verhängt. Die schwächere Wirtschaft Armeniens hätte diese nur schwerlich überstehen können. „Antworten auf Medienfragen zur Situation in Berg-Karabach" (ÜB), Büro des russischen Präsidenten, 17. November 2020, http://kremlin.ru/events/president/news/ 64431.

26 „Telefongespräch von Außenminister Sergej Lavrov mit dem Außenminister der Republik Aserbaidschan, Jeyhun Bayramov" (ÜB), russisches Außenministerium, 15. November 2020, https://www.mi d.ru/en/telefonnye-razgovory-ministra/-/a sset_publisher/KLX3tiYzsCLY/content/id /4434452. 